\title{
Neue Erkenntnisse in Diagnostik und Therapie COPD-Management und COPD-Leitlinien
}

\author{
Prof. Dr. med. C.-P. Criée, Evangelisches Krankenhaus Göttingen-Weende e.V., Göttingen
}

NOTFALLMEDIZIN 2003; 29: 404-408

Die Therapie der COPD richtet sich nach dem Schweregrad. Man unterscheidet eine Risikogruppe und drei Schweregrade der COPD. Als Therapieoptionen stehen Pharmakotherapie, eine nicht-medikamenöse Behandlung und operative Verfahren zur Verfügung. Die Langzeitbehandlung der stabilen COPD ist durch eine schrittweise Erweiterung der Therapieoptionen in Abhängigkeit vom Schweregrad der Erkrankung gekennzeichnet. Treten Exazerbationen auf, muss die Behandlung verändert werden. Etwa die Hälfte der meist leichten Exazerbationen kann ohne ärztlichen Kontakt von den betroffenen Patienten selbst bewältigt werden. Falls im Rahmen der ambulanten Therapie eine Wiederherstellung des Ausgangszustandes vor der Exazerbation nicht gelingt, ist eine stationäre Einweisung notwendig. Die häufigsten Ursachen der Exazerbationen sind virale und/oder bakterielle Atemwegsinfektionen.

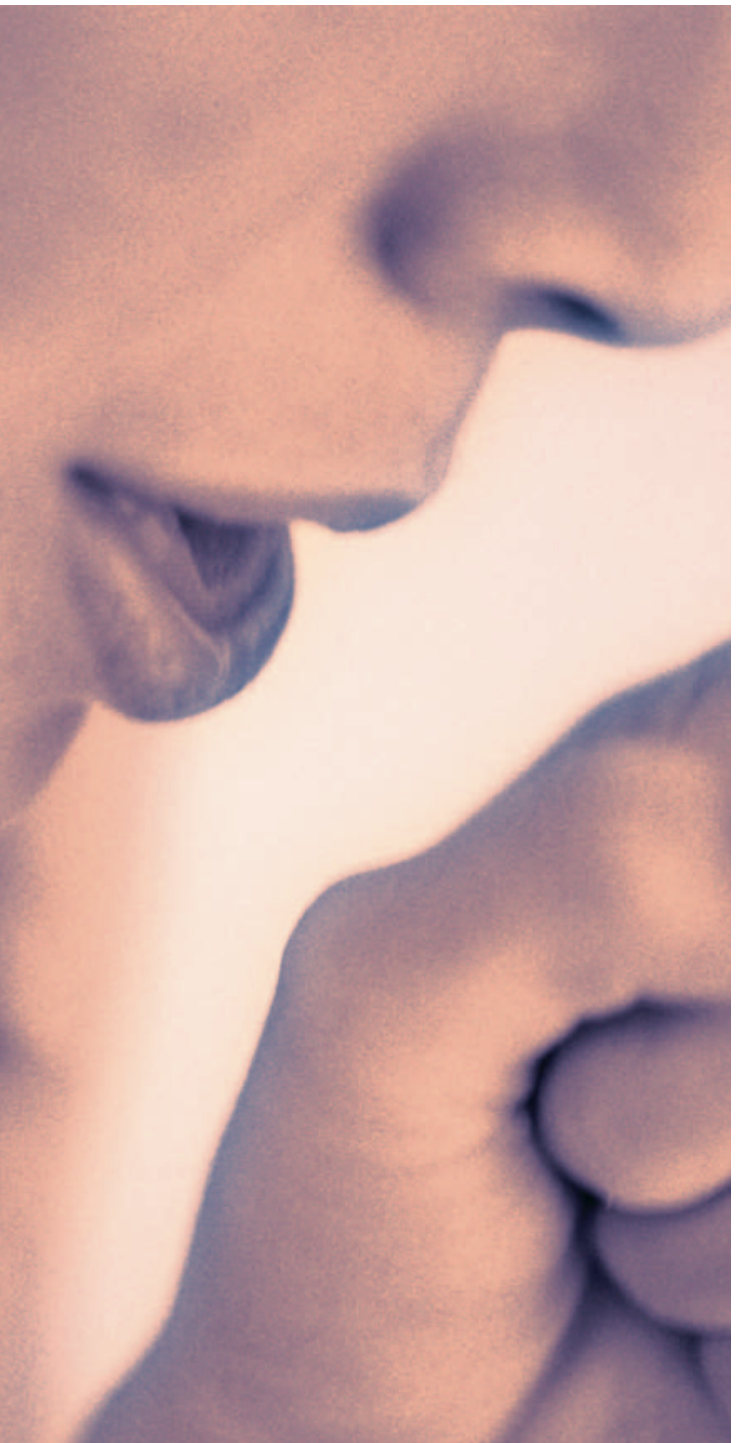

$\mathrm{N}$ eue Erkenntnisse in der Diagnostik und Therapie machten eine Neufassung der Leitlinien der Deutschen Atemwegsliga zur Behandlung von Patienten mit chronisch obstruktiver Bronchitis und Lungenemphysem notwendig, die in der Zeitschrift „Pneumologie“ im letzten Jahr publizierte wurde (1). In Anlehnung an internationale Leitlinien wurde der Begriff COPD (chronic obstructive pulmonary disease) benutzt, der die beiden Krankheiten „chronisch obstruktive Bronchitis“ und „Lungenemphysem“ umfasst. Die COPD lässt sich als eine Krankheit definieren, die durch eine progrediente, nach Gabe von Bronchodilatatoren und/oder Glucocorticoiden nicht vollständig reversible Atemwegsobstruktion auf dem Boden einer chronischen Bronchitis und/oder eines Lungenemphysems gekennzeichnet ist. Sie umfasst daher eine Symptomatik, die durch eine Kombination aus chronischem Husten, gesteigerter Sputumproduktion, Atemnot, Atemwegsobstruktion und eingeschränktem Gasaustausch charakterisiert ist. Nicht eingeschlossen in die Diagnose COPD werden andere Ursachen einer chronischen Atemwegsobstruktion wie Asthma, Mukoviszidose, Bronchiektasien und Bronchiolitis obliterans.

Die Diagnose der COPD wird über die Anamnese, körperliche Untersuchung, Röntgen-Thorax und vor allem durch die Spirometrie und den Bronchospasmolysetest gestellt (Abb. 1). Charakteristisch für Patienten mit COPD ist, dass nach Gabe eines Beta-2-Sympathomimetikums (z.B. $200 \mu \mathrm{g}$ Salbutamol) beziehungsweise eines Anticholinergikums (z.B. $80 \mu \mathrm{g}$ Ipratropiumbromid) 15 bzw. 30 Minuten nach Inhalation die $\mathrm{FEV}_{1}$ um weniger als $15 \%$ des Ausgangswertes und um weniger als $200 \mathrm{ml}$ ansteigt (Reversibilitätstest Bronchodilatator) und dies auch nicht nach Gabe von $40 \mathrm{mg}$ Prednisolonäquivalent/die oder 2000 mg Beclomethasonäquivalent/ die über vier Wochen (Reversibilitätstest, Kortison) der Fall ist.

Verzicht auf Tabakrauchen als effektivste Einzelmaßnahme

Die Therapie der COPD richtet sich nach dem Schweregrad, der wiederum durch Klinik, Symptomatik und vor allem durch die Lungenfunktionsuntersuchung definiert ist. Man unterscheidet eine Risikogruppe und drei Schweregrade der COPD, die in Tabelle 1 dargestellt sind.

Die Langzeitbehandlung der stabilen COPD ist durch eine schrittweise Erweiterung der Therapieoptionen in Abhängigkeit vom Schweregrad der Erkrankung gekennzeichnet (Tab. 2). Der Verzicht auf Tabakrauchen ist die effektivste Einzelmaßnahme zur Reduktion des COPD-Risikos und der Progression der Erkrankung. Das Fortschreiten der Atemwegsobstruktion korreliert mit der täglich konsumierten Zigarettenzahl. Es besteht eine enge Beziehung zwischen Intensität der Beratung zur Aufgabe des Nikotinkonsums und dem Erfolg.

\section{Influenzaschutzimpfung}

Die Influenzaschutzimpfung sollte jährlich bei allen Patienten mit 
chronischer Bronchitis beziehungsweise COPD im Herbst mit der jeweils aktuellen Vakzine durchgeführt werden. Die Influenzaschutzimpfung führt zu einer erheblichen Reduktion der Morbidität, ferner zu einer Abnahme von sekundär auftretenden Pneumonien. Während eine Abnahme der Exazerbationen durch eine Pneumokokken-Schutzimpfung bei Patienten mit COPD nicht gesichert ist, ist die Impfung gegen die bakterielle Form der Pneumokokken-Pneumonie mit hoher Mortalität wirksam.

Die Patientenschulung ist ein wichtiges Therapieelement für alle Schweregrade der Erkrankung, da sie zu einer Steigerung der Effizienz des Managements wesentlich beträgt.

\section{Medikamentöse Therapie}

Die Pharmakotherapie ermöglicht eine Linderung der Beschwerden, eine Besserung der körperlichen Leistungsfähigkeit, der Lebensqualität und/oder eine Reduktion von Exazerbationen, sie kann jedoch nicht die krankheitstypische Verschlechterung der Lungenfunktion verhindern. Bei akuter Atemnot ist die Inhalation kurz wirksamer Beta2-Sympathomimetika mittels Dosieraerosol oder Pulver und/oder die Inhalation von kurz wirksamen Anticholinergika zu empfehlen. Für die Dauertherapie ab dem Schweregrad II ist der regelmäßige Einsatz lang wirksamer Beta-2-Sympathomimetika (Salmeterol, Formoterol) und/ oder lang wirksame Anticholinergika (Tiotropriumbromid) wegen der besseren Handhabung und besserer Effektivität zu empfehlen. Theophyllin ist nur zweite Wahl wegen der geringeren therapeutischen Breite.

Inhalative Glucocorticosteroide sind nur bei Patienten mit COPD und einer asthmatischen Komponente indiziert, etwa bei Vorliegen einer bronchialen Hyperreaktivität mit einem Anstieg der Sekundenkapazität $\left(\mathrm{FEV}_{1}\right)$ um mehr als $15 \%$ beziehungsweise $200 \mathrm{ml}$ gegenüber dem Ausgangswert nach etwa 4-wöchiger Applikation von Glucocorticosteroiden (Reversibilitätsteste, siehe Abb. 1). Bei symptomatischen COPD-Patienten der Schweregrade II und III

\section{Abb. 1 Flussdiagramm zur Diagnostik der COPD}

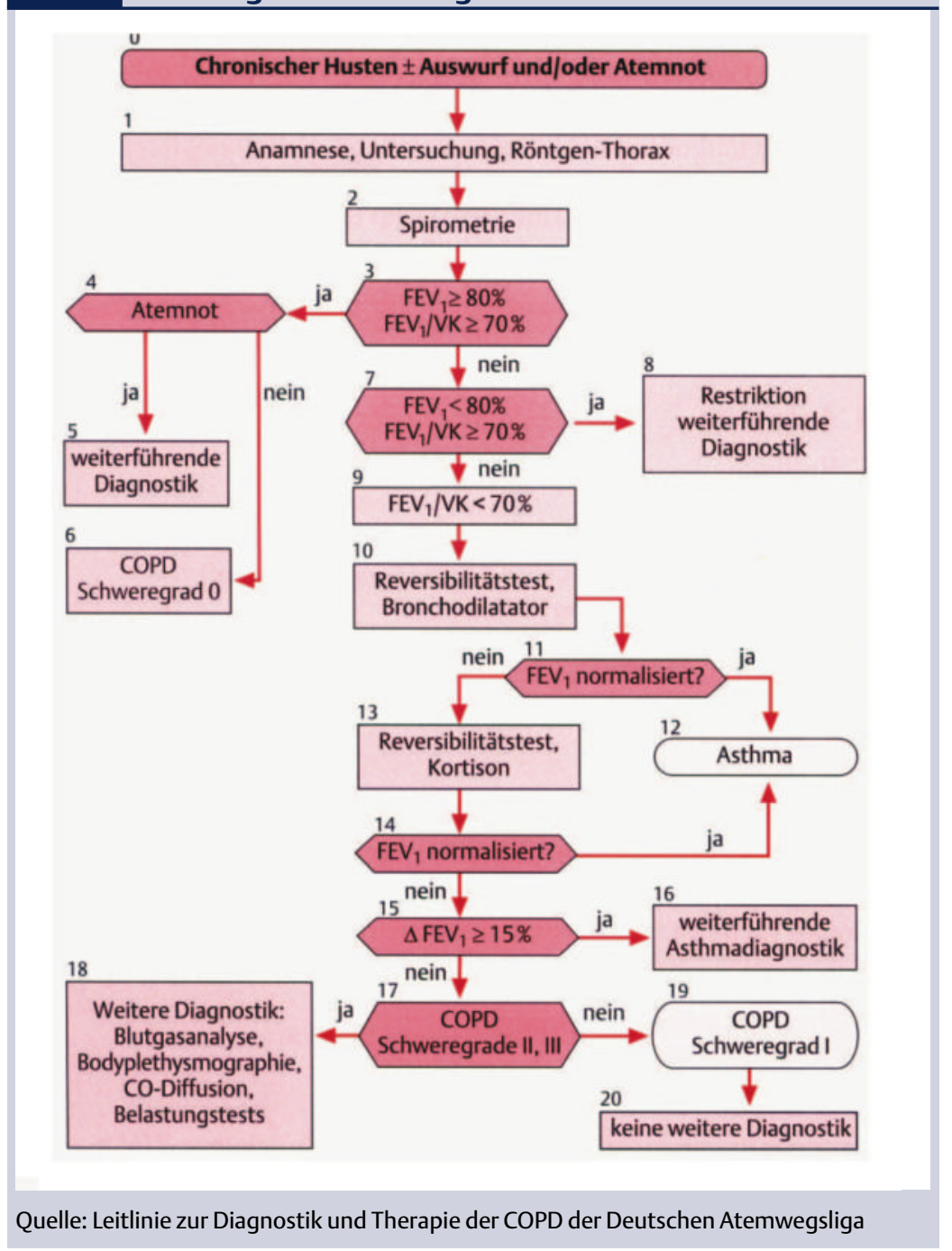

ist eine Dauertherapie mit inhalativen Corticosteroiden indiziert, wenn sich die Lungenfunktion und/oder die klinische Symptomatik während eines Behandlungsversuches über drei Monate bessert. Nach internationalen Empfehlungen können auch inhalative Steroide bei Patienten mit einer $\mathrm{FEV}_{1}<50 \%$ des Solls mit immer wiederkehrenden Exazerbationen gegeben werden (3). Eine Langzeittherapie mit oralen Glucocorticosteroiden wird bei der COPD nicht empfohlen! Eine Kombination aus lang wirksamen Beta-2Sympathomimetika und Glucocorticosteroiden ist dann indiziert, wenn beide Einzelsubstanzen idiziert sind.

Die Indikation zum Einsatz von Mukopharmaka zur besseren Sekretelimination sollte kritisch gestellt werden und sich an dem subjektiven
Therapieerfolg orientieren. Eine generelle Anwendung von Immunmodulatoren (lyophilisierter normierter Bakterienextrakt) kann derzeit nicht generell empfohlen werden. Antitussiva sind bei quälendem trockenen Husten im Rahmen von Exazerbationen hilfreich falls keine Hyperkapnie vorliegt und keine Atemdepression droht.

\section{Nichtmedikamentöse Therapie}

In der Langzeitbehandlung ist die nichtmedikamentöse Therapie ebenso wichtig wie die medikamentöse Therapie:

\section{Ernährung}

Die Unterernährung verschlechtert die Prognose. Ziel der Ernährungstherapie ist es, mittels oraler 


\section{Tab. 1 Schweregradeinteilung der COPD (modifiziert nach GOLD ${ }^{1}$ )}

\begin{tabular}{|c|c|}
\hline $\begin{array}{l}\text { Schweregrad } \\
\text { III (schwer) }\end{array}$ & $\begin{array}{l}\text { Charakteristik } \\
\text { - } \text { FEV }_{1}<30 \% \text { Soll, FEV } / \text { VK }<70 \% \\
\text { oder } \\
\text { - } \text { FEV }_{1}<50 \% \text { Soll und chronische } \\
\text { respiratorische Insuffizienz oder } \\
\text { Rechtsherzinsuffizienz }\end{array}$ \\
\hline II (mittelgradig) & $\begin{array}{l}-30 \% \leq \mathrm{FEV}_{1}<80 \% \text { Soll, } \mathrm{FEV}_{1} / \mathrm{VK} \\
<70 \% \\
\text { - mit/ohne chronische Sympto- } \\
\text { me (Husten, Auswurf, Dyspnoe) }\end{array}$ \\
\hline I (leichtgradig) & $\begin{array}{l}\text { - } \mathrm{FEV}_{1} \geq 80 \% \text { Soll, } \mathrm{FEV}_{1} / \mathrm{VK}<70 \% \\
\text { - mit/ohne Symptomatik (Hus- } \\
\text { ten, Auswurf, Dyspnoe evtl. bei } \\
\text { starker körperlicher Belastung) }\end{array}$ \\
\hline 0 (Risikogruppe) & $\begin{array}{l}\text { - normale Spirometrie } \\
\text { - chronische Symptome (Husten, } \\
\text { Auswurf) }\end{array}$ \\
\hline \multicolumn{2}{|c|}{${ }^{1}$ Global Initiative for Obstructive Lung Disease } \\
\hline $\begin{array}{l}\text { Quelle: Leitlinie zu } \\
\text { Deutschen Atemn }\end{array}$ & $\begin{array}{l}\text { agnostik und Therapie der COPD } \\
\text { liga }\end{array}$ \\
\hline
\end{tabular}

Die Schweregradeinteilung erfolgt anhand der nach akuter Bronchodilatation gemessenen FEV $_{1}$ Werte (\% vom Soll) bei stabiler COPD.

Kalorienzufuhr, gegebenenfalls Ernährungssupplementierung, eine Gewichtszunahme zu erzielen.

\section{Physiotherapie}

- Senkung der Atemarbeit: Lippenbremse, kleine Geräte mit einer exspiratorisch wirksamen Stenose.

- Atemerleichternde Körperstellungen bei schwerer Dyspnoe: Kutschersitz, Anlehnen an eine Wand.

- Verbesserung der Sekretlimitation: Lagerungsdrainage, effektive Hustentechniken und die Mobilisierung des Sekrets mittels Thoraxwandperkussion und Geräten mit exspiratorischen Oszillationen (Flutter, RC Cornet).

\section{Patientenschulung}

Empfehlenswert in kleinen Gruppen von maximal 4-8 Teilnehmern mit strukturierten und evaluierten Programmen.

\section{Rehabilitation}

Linderung der somatischen und psychischen Symptomatik, Steigerung der Lebensqualität mit Wiederherstellung der bestmöglichen Leis- tungsfähigkeit, Förderung der sozialen Reintegration. Rehabilitation erfolgt stationär, teilstationär und/ oder wohnortnah ambulant.

\section{Langzeit- Sauerstofftherapie}

Die Langzeit-Sauerstofftherapie (LOT) ist bei chronischer Hypoxämie (arterieller Sauerstoffpartialdruck $\leq$ $55 \mathrm{mmHg}$ ) indiziert; bei Cor pulmonale bereits bei einem $\mathrm{pO}_{2}<60$ $\mathrm{mmHg}$. Anwendungsdauer: mindestens 16 Stunden pro Tag.

\section{Heimbeatmung}

Eine Entlastung der Atemmuskulatur durch die intermittierende (nächtliche) nichtinvasive Heimbeatmung über Nasen- oder NasenMund-Masken bei ventilatorischer Insuffizienz ist indiziert, wenn alle konservativen Behandlungsmöglichkeiten ausgeschöpft sind und der Patient weiterhin hyperkapnisch ist. Einleitung nur in spezialisierten Krankenhausabteilungen.

\section{Operative Therapieverfahren Bullektomie}

Die Bullektomie wird bei großen Bullae angewandt, die mehr als $1 / 3$ eines Lungenflügels einnehmen und das benachbarte Lungenparenchym komprimieren.

\section{Lungenvolumenreduktion}

Die Resektion von stark emphysematös veränderten Lungenarealen, vor allem in den Oberfeldern, bei sorgfältig ausgewählten, geeigneten Patienten in spezialisierten Zentren.

\section{Lungentransplantation}

Für Patienten mit schwerem Lungenemphysem. Eine immunsuppressive Therapie ist lebenslang erforderlich. Die Auswahl der Patienten erfolgt durch spezialisierte Zentren.

\section{Exazerbationen bei COPD}

Eine Exazerbation ist definiert als eine Zunahme von Symptomen (Atemnot, Husten, Auswurf, Obstruktion, thorakale Beklemmung, selten Fieber), die eine Änderung der Behandlung erforderlich macht. Akute Verschlechterungen treten bei COPD-Patienten häufig auf. Während zirka 50\% der meist leichten Exazerbationen ohne ärztlichen Kontakt von den betroffenen Patienten selbst bewältigt werden, liegt die Krankenhaussterblichkeit bei schweren Exazerbationen zwischen 3 und 10\%. Bei Beatmung auf der Intensivstation liegt die Sterblichkeit bei 20-30\%. Die häufigsten Ursachen der Exazerbationen sind virale und/oder bakterielle Atemwegsinfektionen. Wichtig bei der Diagnostik ist eine Röntgenaufnahme des Thorax in zwei Ebenen bei Verdacht auf eine Pneumonie, eine Blutgasanalyse, eine Lungenfunktionsuntersuchung mit Bestimmung der $\mathrm{FEV}_{1}$ (soweit es der Zustand erlaubt). Eine mikrobiologische Sputumdiag-

\section{Tab. 2 Langzeittherapie der stabilen COPD}

\begin{tabular}{|c|c|c|}
\hline $\begin{array}{l}\text { Schweregrad } \\
\text { Alle (incl. } \\
\text { Risikogruppe) }\end{array}$ & $\begin{array}{l}\text { Medikamente } \\
\text { - }\end{array}$ & $\begin{array}{l}\text { Nichtmedikamentöse Therapie } \\
\text { Risikofaktoren meiden, } \\
\text { Patientenschulung, } \\
\text { Schutzimpfungen }\end{array}$ \\
\hline III & $\begin{array}{l}\text { zusätzlich prüfen, ob eine } \\
\text { Langzeit- } \mathrm{O}_{2} \text {-Therapie } \\
\text { angezeigt ist }\end{array}$ & $\begin{array}{l}\text { Rehabilitation (evtl. primär } \\
\text { stationär), adäquate Ernährung } \\
\text { Weitere Möglichkeiten: Heim- } \\
\text { beatmung, Emphysemchirurgie }\end{array}$ \\
\hline $\begin{array}{c}\uparrow \\
\text { Bei fehlender } \\
\text { Besserung } \\
\uparrow\end{array}$ & $\begin{array}{l}\text { Therapieversuch mit ICS über } \\
3 \text { Monate, bei Ansprechen } \\
\text { Fortsetzung } \\
\text { zusätzlich Theophyllin } \\
\text { B2S und/oder (ggf. kombi- } \\
\text { niert) Anticholinergika }\end{array}$ & $\begin{array}{l}\text { Körperliches Training. } \\
\text { Physiotherapie, } \\
\text { adäquate Ernährung }\end{array}$ \\
\hline I & $\begin{array}{l}\text { B2S und/oder } \\
\text { Anticholinergika bei Bedarf }\end{array}$ & \\
\hline Risikogruppe & Keine Medikation & \\
\hline
\end{tabular}




\section{Abb. 2 Ambulantes Management der COPD-Exazerbation}

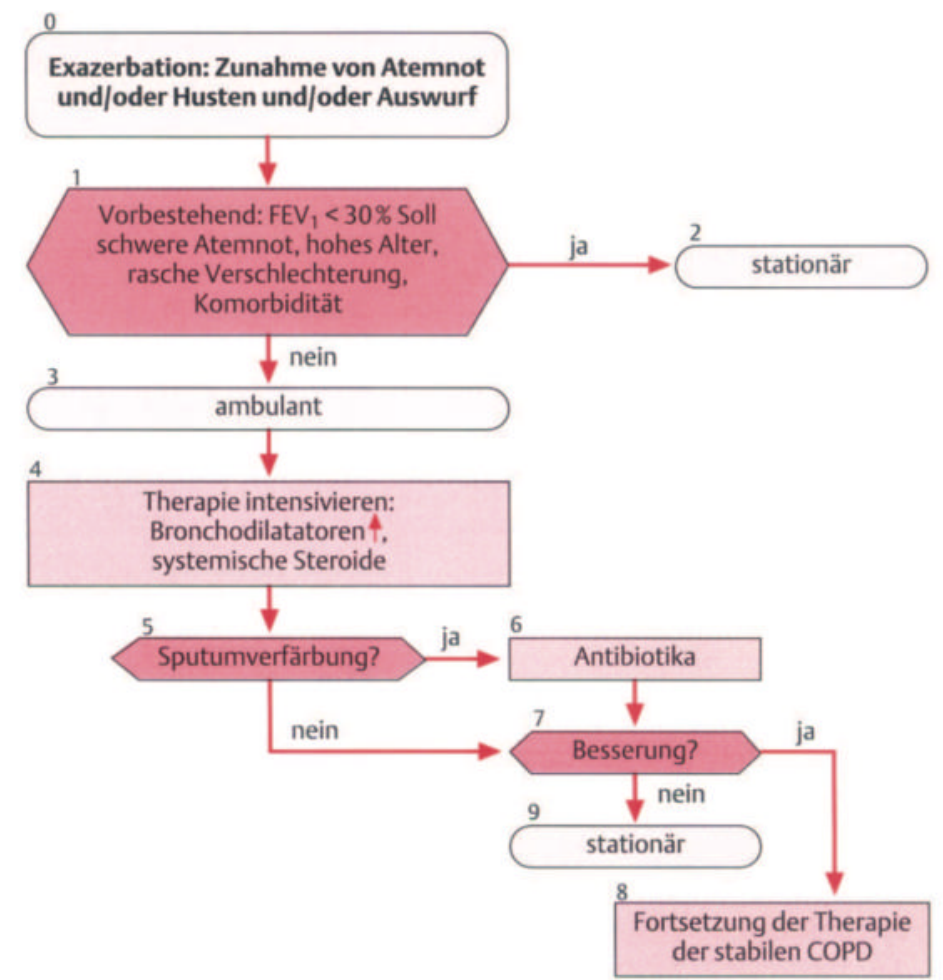

Quelle: Leitlinie zur Diagnostik und Therapie der COPD der Deutschen Atemwegsliga

nostik ist nur bei Patienten mit schwerer COPD und häufigen Exazerbationen notwendig.

Prinzipien des ambulanten Managements (Abb. 2)

Medikamentös sollte die bronchodilatatorische Therapie intensiviert werden: Initial 1-2 Hübe eines kurz wirksamen Beta-2-Sympathomimetikums +2 Hübe eines kurz wirksamen Anticholinergikums alle 10 bis 15 Minuten; bei unzureichender Besserung:

- 1,0-2,5 mg eines $\beta_{2}$-Adrenergikums + 0,5 mg Ipratropiumbromid in einen Vernebler füllen und mindestens 10 Atemzüge inhalieren lassen, alle 10-15 Minuten.

- $\quad \beta_{2}$-Adrenergika können alternativ i.v. (Reproterol $90 \mu$ g) oder s.c. (250-500 $\mu \mathrm{g}$ Terbutalin) gespritzt werden.

- Theophyllin als „second line“ Medikation: Bolus 200 mg (Injektionsdauer 5 Minuten) gegebenenfalls anschließend als Dauerinfusion (Anfangsdosis
$800 \mathrm{mg} / 24 \mathrm{~h})$ je nach Theophyllin-Serumspiegel (8-20 mg/L). Bei Theophyllin-Vorbehandlung kein Bolus.

- Systemische Glucocorticoide: 20-40 mg Prednisolonäquivalent/die, maximal für 14 Tage (längere Behandlung bringt keine Vorteile).

- Antibiotika: wenn ein bakterieller Infekt mit purulenter Verfärbung des Sputums vorliegt. Kalkulierte Antibiose: S. pneumoniae, H. influenzae, B. catarrhalis erfassen, bei hochgradiger Obstruktion $\left(\mathrm{FEV}_{1}<30 \%\right.$ Soll) auch Pseudomonas aeruginosa und gramnegative Enterobakterien (Aminopenicillin \pm ß-Laktamaseinhibitor, Oralcephalosporine, Makrolide; bei problematischer Resistenzlage auch Ketolide oder Fluorochinolone der Klassen 3 und 4). Therapiedauer: 5-7, in schweren Fällen etwa 10 Tage, gegebenenfalls intravenös einzuleiten. Bei Misserfolg: mikrobiologische Diagnostik nach 2-3 Tagen Antibiotikapause.
Sind Diuretika bei Rechtsherzinsuffizienz indiziert, initial $40 \mathrm{mg}$ Furosemid i.v..

\section{Stationäre Therapie bei \\ Exazerbation}

Falls im Rahmen der ambulanten Therapie eine Wiederherstellung des Ausgangszustandes vor der Exazerbation nicht gelingt, beziehungsweise die Exazerbation progredient verläuft oder weitere Maßnahmen wie Sauerstoffgabe oder eine nichtinvasive Beatmung erforderlich werden sollte, ist eine stationäre Einweisung notwendig. Im Krankenhaus sollte bei respiratorischer Insuffizienz sofort die Sauerstoffgabe über eine Nasensonde beziehungsweise Atemmaske eingeleitet werden. Ziel der Sauerstofftherapie ist eine adäquate Oxygenierung mit arteriellen $\mathrm{PO}_{2}$-Werten von mehr als $60 \mathrm{mmHg}$ beziehungsweise einer $\mathrm{O}_{2}$-Sättigung von über $90 \%$. Eine Kontrolle zur Beurteilung des Erfolges und zur Überprüfung einer unter $\mathrm{O}_{2}$-Gabe auftretenden $\mathrm{CO}_{2}$-Retention kann bereits nach 20 Minuten durchgeführt werden und sollte im Verlauf immer wieder überprüft werden.

Bei immobilen Patienten, bei Patienten mit Polyglobulie, Dehydration, bei Zeichen der chronisch venösen Insuffizienz und/oder thromboembolischen Erkrankungen sollte eine Heparinisierung erfolgen, wobei niedermolekulare wie Standardheparine einsetzbar sind. Bei großen Sputummengen und lobären Atelektasen sollte auf jeden Fall bronchoskopisch abgesaugt werden. Weitere Maßnahmen sind Physiotherapie mit Lagerungsdrainagen sowie Einsatz von Hilfsmitteln mit und ohne Oszillationen und nachfolgendem Huffing.

Die nichtinvasive Beatmung mit positivem Druck (NIV) mit Nasenoder Nasen-Mund-Masken bei akuter respiratorischer Insuffizienz von COPD-Patienten wurde in den letzten Jahren in zahlreichen unkontrollierten und fünf randomisierten kontrollierten Studien geprüft. Die NIV führte zu einer Reduktion der respiratorischen Azidose, einem Abfall des $\mathrm{PaCO}_{2}$ sowie einer Abnahme der Atemnot und zu einer Senkung der Mortalität um 50\%! $(4,5)$ Wich- 


\section{Abb. 3 Stationäre Therapie der COPD-Exazerbation}

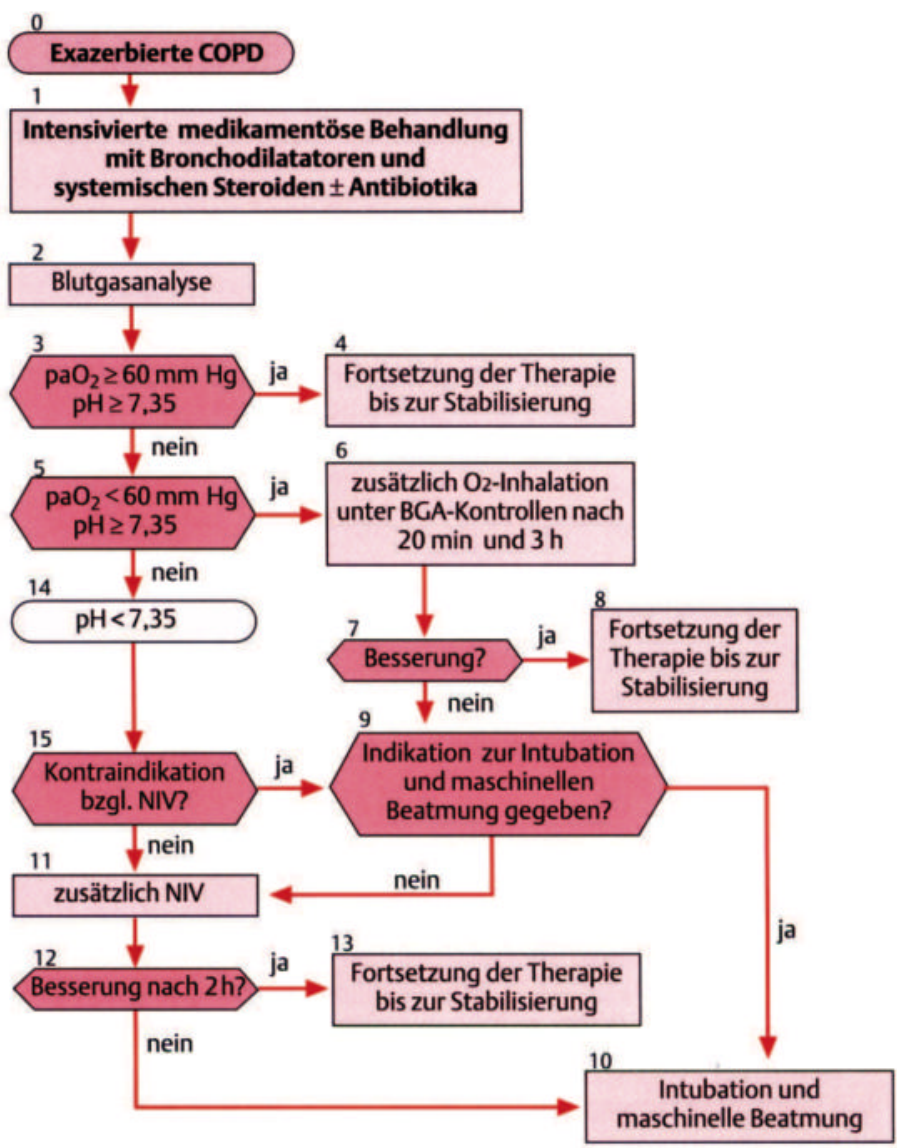

Quelle: Leitlinie zur Diagnostik und Therapie der COPD der Deutschen Atemwegsliga

tigster Effekt der NIV ist die Verhinderung der Intubation.

Die stationäre Therapie der Exazerbation der COPD einschließlich Sauerstoffgabe und nichtinvasiver Beatmung (NIV) ist in Abbildung 3 aufgeführt. Grundsätzlich gilt, dass die nichtinvasive Beatmung umso häufiger erfolgreich durchgeführt werden kann, je geringer die hyperkapnische Azidose ist und je kooperationsfähiger der Patient ist. Wenn die Hyperkapnie zu einem geringeren $\mathrm{pH}$ als 7,2 führt, ist die Erfolgschance einer nichtinvasiven Beatmung gering. Daher sollten Patienten mit COPD frühzeitig zur stationären Behandlung eingewiesen werden um eine nichtinvasive Beatmung durchführen zu können, sobald bei Hyperkapnie der pH 7,35 unterschritten wird. Durch die nichtinvasive Beatmung kann die Intensivstation vermieden werden und die Mortalität um 50\% gesenkt werden! Wenn die nichtinvasive Beatmung erfolglos ist, ist die invasive Beatmung indiziert. Bei COPDPatienten mit invasiver Beatmung ist mit einer deutlichen Verlängerung des Aufenthaltes auf der Intensivstation zu rechnen, die Mortalität der maschinell beatmeten Patienten liegt zwischen 20 und 30\%. Die Entwöhnung von invasiver Beatmung gestaltet sich bei COPD-Patienten häufig sehr schwierig. Es sollte daher nach Überwindung der akut lebensbedrohlichen Situationen möglichst schnell eine Umstellung der invasiven Beatmung auf die nichtinvasive Beatmung unter Vermeidung der Tracheostomie erfolgen, damit der Patient dann über die nichtinvasive Beatmung entwöhnt werden kann beziehungsweise mit einer intermittierenden nichtinvasiven Beatmung in die Heimbeatmung nach Hause entlassen werden kann.

\section{Summary}

Treatment of COPD is dictated by the degree of its severity. A risk group and three degrees of severity of COPD are differentiated. Available treatment options include pharmacotherapy, non-medical treatment and surgical procedures. Long-term treatment of COPD is characterised by a stepwise extension of therapeutic options as a function of the severity of the condition. Should there be exacerbations, the treatment must be altered. About one-half of the usually mild - exacerbations can be dealt with by the patient himself, without the need to visit the doctor. If, during the course of ambulatory treatment, restoration to the preexacerbation state is not successful, hospitalisation is indicated. The most common causes of exacerbations are viral and/or bacterial airway infections

\section{Literatur}

1. Worth H, Buhl R, Cegla U, Criée C-P et al. Leitlinie der Deutschen Atemwegsliga und der Deutschen Gesellschaft für Pneumologie zur Diagnostik und Therapie von Patienten mit chronisch obstruktiver Bronchitis und Lungenemphysem (COPD). Pneumologie 2002; 56: 704-738

2. Kardos P. Leitlinie zur Diagnostik und Therapie der COPD. Hrsg. von der Deutschen Atemwegsliga. Stuttgart-New York: Georg Thieme Verlag, 2002

3. Pauwels RA, Buist AS, Calverley PMA et al. Global strategy for the diagnosis, management, and prevention of COPD. Am. J. Respir. Crit. Care Med 2001; 163: 12561276

4. Brochard L, Mancebo J, Wysock M et al. Noninvasive ventilation for acute exacerbations of chronic obstructive pulmonary disease. N. Engl. J. Med. 1995; 33: 817-822 5. Plant PK, Owen JL, Elliott MW. Early use of non-invasive ventilation (NIV) for acute exacerbations of COPD on general respiratory wards: a multicenter randomized controlled trial. Lancet 2000; 355: $1931-$ 1935

\section{Anschrift des Verfassers}

Prof. Dr. med. Carl-Peter Criée Pneumologie, Beatmungsmedizin/ Schlaflabor

Evangelisches Krankenhaus Göttingen-Weende e.V. 37120 Bovenden-Lenglern 